\title{
PULMONARY MANIFESTATION OF LYMPHANGIOLEIOMYOMATOSIS: A CASE REPORT
}

\section{B. K. Duara1 ${ }^{1}$ Debjanee Phukan ${ }^{2}$,Himangshu Sarma ${ }^{3}$, Rohit Chandak ${ }^{4}$, Romita Das ${ }^{5}$}

1 Professor and HOD, Department of Radiology, Gauhati Medical College and Hospital, Guwahati, Assam. 2Junior Resident, Department of Radiology, Gauhati Medical College and Hospital, Guwahati, Assam. 3 Junior Resident, Department of Radiology, Gauhati Medical College and Hospital, Guwahati, Assam. 4 Junior Resident, Department of Radiology, Gauhati Medical College and Hospital, Guwahati, Assam. 5Junior Resident, Department of Radiology, Gauhati Medical College and Hospital, Guwahati, Assam.

\section{ABSTRACT}

Lymphangioleiomyomatosis (LAM) is an uncommon interstitial lung disease that exclusively affects women, usually during their reproductive years. LAM is characterized pathologically by abnormal proliferation of LAM cells in the lungs and in thoracic and retroperitoneal lymphatics. ${ }^{1}$ We presented a case of 40 -year-old female presented to Department of Pulmonary Medicine, Gauhati Medical College with cough and chest pain for 6 months, which was diagnosed to be pulmonary manifestation of Lymphangioleiomyomatosis in HRCT chest.

\section{KEYWORDS}

High Resolution Computed Tomography, Lymphangioleiomyomatosis (LAM), Pulmonary Cysts, Lung Parenchyma.

HOW TO CITE THIS ARTICLE: Duara BK, Phukan D, Sarma H, et al. Pulmonary manifestation of lymphangioleiomyomatosis: a case report. J. Evolution Med. Dent. Sci. 2016;5(40):2488-2490, DOI: 10.14260/jemds/2016/580

\section{INTRODUCTION}

Lymphangioleiomyomatosis (LAM) is a rare, idiopathic disorder that predominantly affects the lung parenchyma of women of childbearing age. It is characterized by pulmonary cysts at Computed Tomography (CT). The radiologist is often the first to suggest the diagnosis of LAM. ${ }^{1}$ A small percentage of patients, also typically women have LAM in association with Tuberous Sclerosis Complex (TSC). LAM is characterized by the abnormal proliferation of smooth muscle cells (LAM cells) in the lungs and in the thoracic and retroperitoneal lymphatics. Affected patients are at risk of developing renal hamartomas or angiomyolipomas. Patients with LAM characteristically present with chronic dyspnoea and cough and less commonly with spontaneous pneumothorax. ${ }^{2}$

We present here a case of lymphangioleiomyomatosis in a 40-year-old female patient. We also review the imaging, clinical and pathologic aspects of this unusual disease entity.

\section{CASE PRESENTATION}

A 40-year-old female presented to the Department of Pulmonary Medicine in the month of October 2015 with cough and occasional difficulty in breathing for 6 months. There was no associated fever. There is no history of epilepsy. On examination of the patient, no facial rashes noted. Chest X-ray done, which shows reticular opacity in bilateral lung fields. On HRCT examination, we found multiple variable sized smooth walled cystic lesion diffusely scattered in bilateral lung field with involvement of all the lobes with intervening normal lung parenchyma. There are features of pulmonary hypertension in the form of increased diameter of pulmonary artery measuring up to $32 \mathrm{~mm}$. Both the kidney appears normal without any evidence of focal lesion in NECT abdomen.

Financial or Other, Competing Interest: None.

Submission 19-02-2016, Peer Review 08-04-2016,

Acceptance 13-04-2016, Published 19-05-2016.

Corresponding Author:

Dr. Debjanee Phukan,

PG Girls Hostel-6,

Gauhati Medical College and Hospital,

Bhangagarh,

Guwahati-781032,

Assam.

E-mail: debjaneephukan@gmail.com

DOI: $10.14260 /$ jemds/2016/580

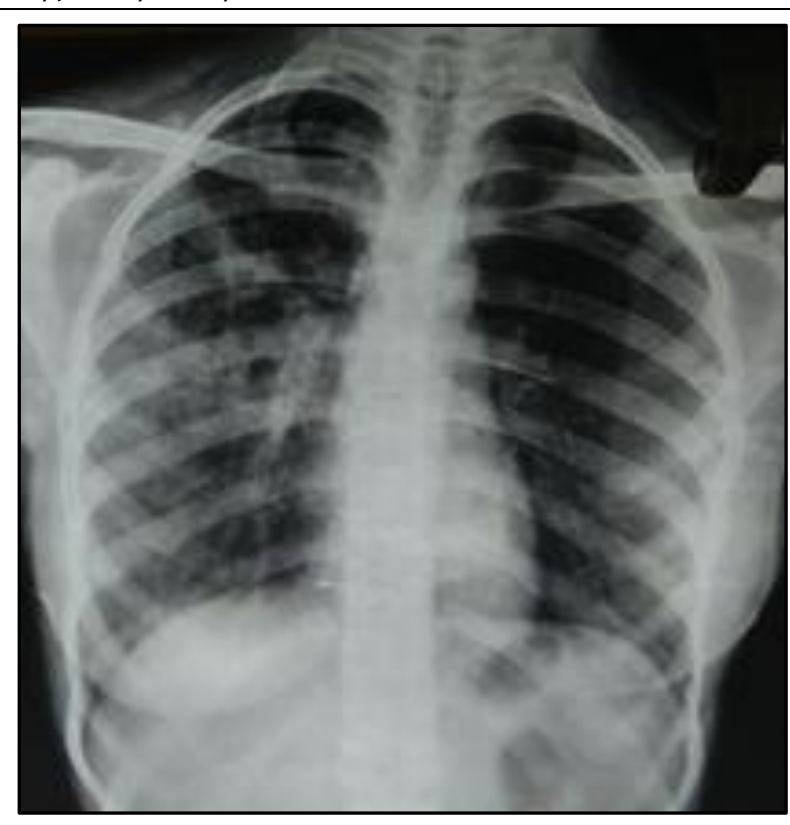

Fig. 1: Chest X-ray showing Reticular Opacities in Bilateral Lung Fields

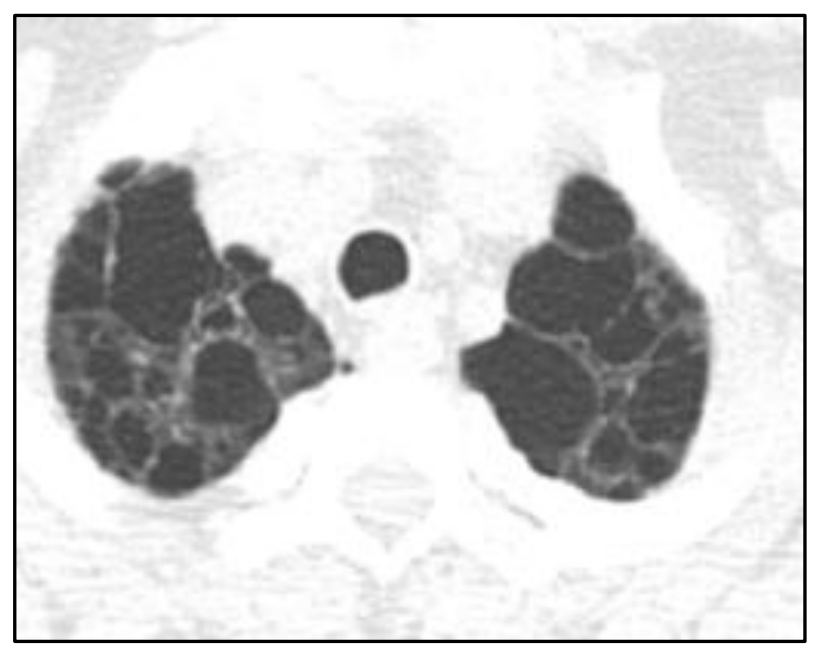

Fig. 2: HRCT of Lung at the Level of Apex showing Multiple Cysts in Bilateral Lung Parenchyma 


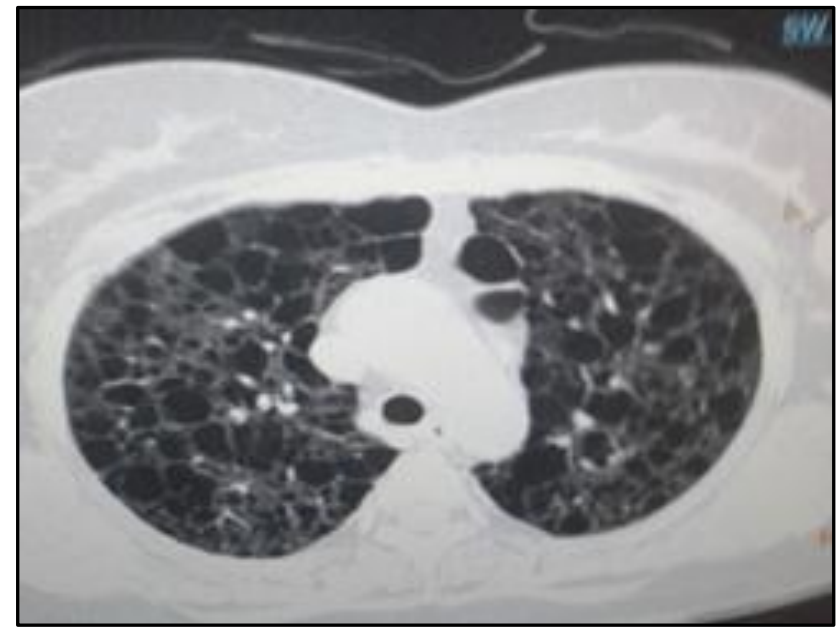

Fig. 3: Axial Section at the Level of Arch of Aorta showing Multiple Cysts in Bilateral Lung Parenchyma

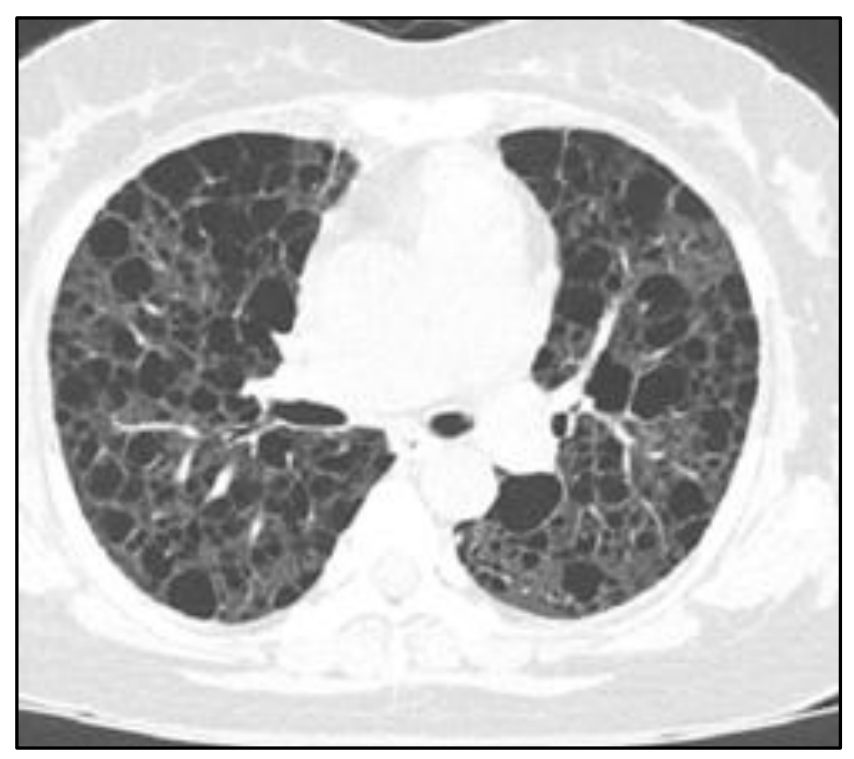

Fig. 4: Axial Section HRCT at the Level Heart showing Multiple Cysts in Bilateral Lung Parenchyma

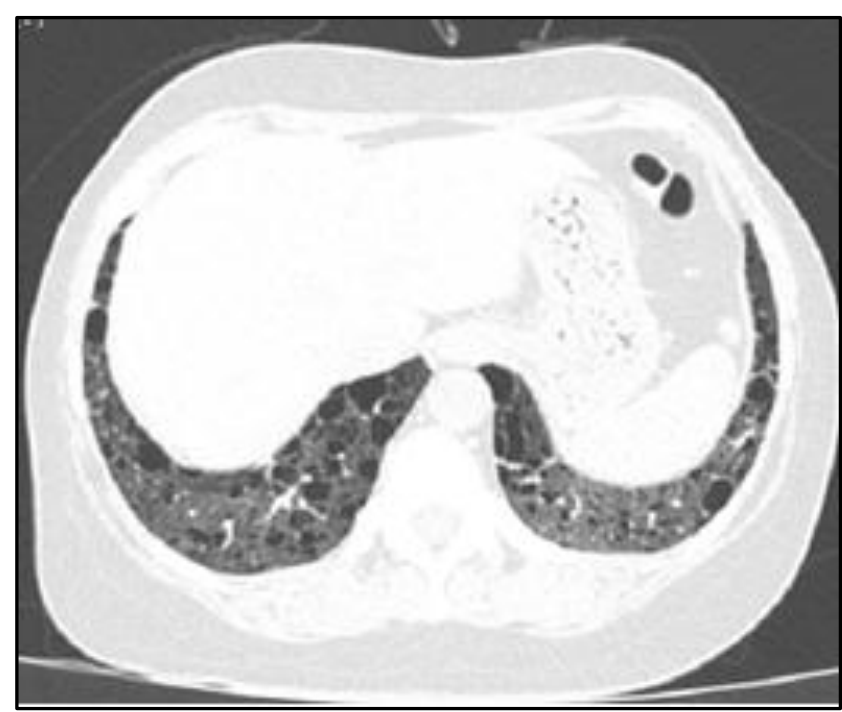

Fig. 5: Axial Section HRCT at the Level Lung Bases showing Multiple Cysts in Bilateral Lung Parenchyma

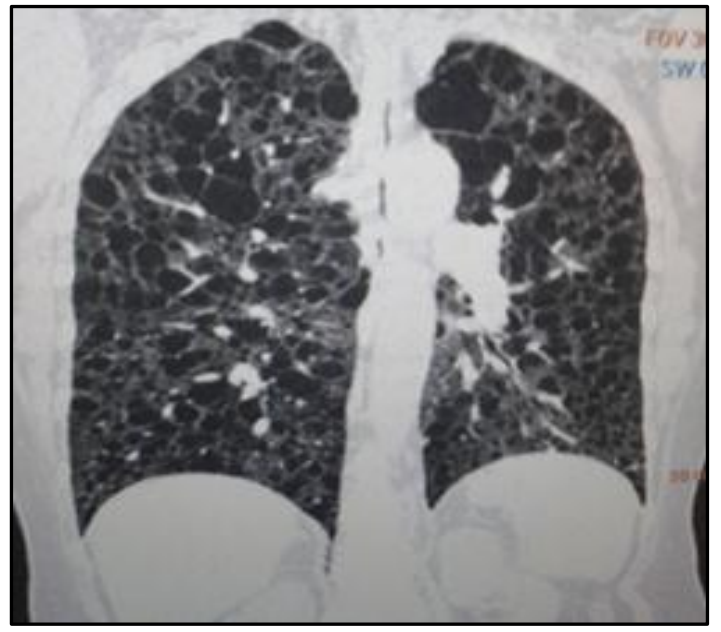

Fig. 6: Showing Multiple Variable Sized Cystic Lesion Occupying Bilateral Lung Fields

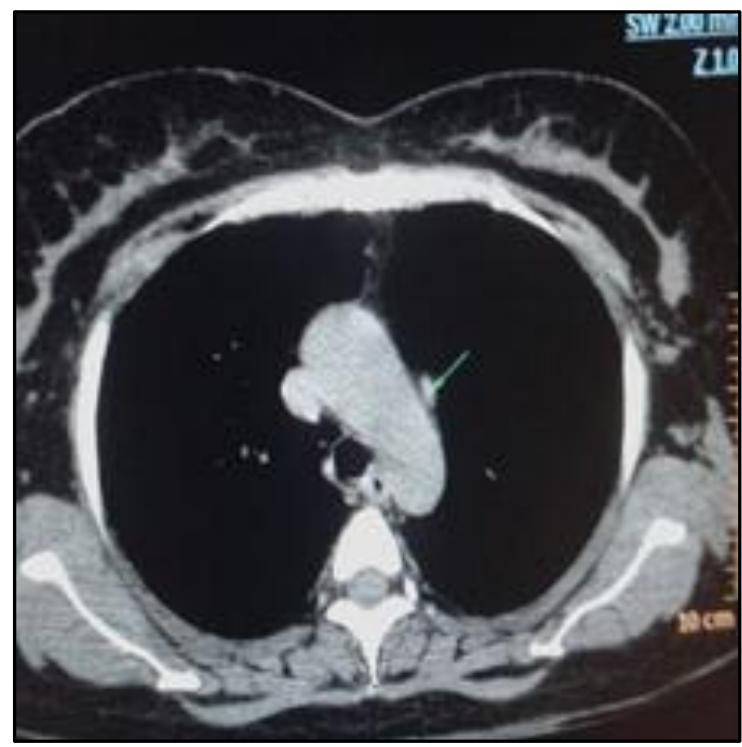

Fig. 7: Mediastinal Window showing Few Prominent Nodes in Prevascular Stations

\section{DISCUSSION}

In 1918, Lutembacher published what is considered the first report of cystic lung disease occurring in association with TSC in a 36-year-old woman with bilateral pneumothoraces who died. Lutembacher attributed the pulmonary nodules and cysts found in this patient to metastases from renal fibrosarcoma. $^{2}$

\section{Clinical Features Demographics}

LAM occurs almost exclusively in women. The average age at onset of symptoms is approximately 34 years. During the course of the illness, there may be non-productive cough, haemoptysis, chylous pleural effusion or chylous ascites. Obstruction of pulmonary venules causes vascular congestion and haemoptysis and lymphatic obstruction leads to chylothorax and chylous ascites. ${ }^{1}$

\section{Imaging Features Chest Radiography}

The radiologist is often the first to suggest the diagnosis of LAM. However, misdiagnosis is common and may result in inappropriate therapeutic procedures that can further 
complicate treatment. ${ }^{1}$ Radiographic abnormalities in patients with LAM have been reported as reticular, reticulonodular, and miliary opacities or pulmonary cysts. These manifestations may precede, accompany or postdate other thoracic manifestations of the disease including pneumothorax and chylous pleural effusion. The most commonly described radiographic manifestation of LAM is a pattern of generalized, symmetric, reticular or reticulonodular opacities seen in approximately $80 \%-90 \%$ of affected patients. In general, radiographic abnormalities have been reported as varying roughly according to disease severity, with more apparent reticulation and cystic changes described in patients with severe pulmonary involvement.

\section{Computed Tomography}

Pulmonary cysts have been present in nearly all cases of LAM reported to date. The mechanism of cyst formation in patients with LAM is controversial, both small airway obstruction and proteolytic destruction with amalgamation of alveoli have been proposed as possible mechanisms. High-spatialresolution CT is both sensitive and specific in the diagnosis of pulmonary LAM, depicting characteristic round thin-walled cysts uniformly distributed throughout the lungs. ${ }^{3}$

The CT manifestations of LAM are distinctive, characterized by numerous thin-walled cysts surrounded by normal lung parenchyma and distributed diffusely and bilaterally. Conventional CT and high-resolution CT findings are almost always abnormal at the time of diagnosis and typically demonstrate parenchymal cysts, even when the chest radiographs appear normal or reveal only pleural effusion or pneumothorax. The cysts are generally symmetrically and uniformly distributed throughout the lungs affecting the upper and lower and the central and peripheral lung parenchyma. ${ }^{4}$ The lung parenchyma between the cysts is typically normal, but in highly cellular forms with significant smooth cell proliferation, small nodules, reticulation or ground-glass attenuation may appear. ${ }^{1}$

The profusion of cysts and the quantitative CT scores clearly correlate with impairment on functional tests and clinical conditions.
CT was more accurate than chest radiography in defining the presence and extent of parenchymal cysts and provided for greater morphologic-physiologic correlation. CT, particularly high-resolution CT, may be useful in the diagnosis and longitudinal evaluation of patients with this disease and may be more sensitive than pulmonary function tests in the early stages of lung damage. ${ }^{4}$

\section{CONCLUSION}

LAM is an uncommon interstitial lung disease that exclusively affects women usually during their reproductive years. Evaluation of pulmonary cyst in lymphangioleiomyomatosis is best done with CT. Chest radiography often fail to demonstrate or may lead to underestimation of the extent of the disease. 5 We presented a case of lymphangioleiomyomatosis in a 40 year-old female patient. We recognize the condition only after HRCT examination.

\section{REFERENCES}

1. Esther Pallisa, Pilar Sanz, Antonio Roman, et al. Lymphangioleiomyomatosis: pulmonary and abdominal findings with pathologic correlation. Radio Graphics 2002;22(1):S185-98.

2. Gerald F Abbott, Melissa L Rosado-de-Christenson, Aletta Ann Frazier, et al. Lymphangioleiomyomatosis: radiologic-pathologic correlation. Radio Graphics 2005;25(3):803-28.

3. Anil K Attili, Ella A Kazerooni. Lymphangioleiomyomatosis. Radiology 2007;244(1):303-8.

4. Aberle DR, Hansell DM, Brown $K$, et al. Lymphangioleiomyomatosis: CT, chest radiographic, and functional orrelations. Radiology 1990;176(2):335-9.

5. Lenoir S, Grenier P, Brauner MW, et al. Pulmonary lymphangioleiomyomatosis and tuberous sclerosis: comparison of radiographic and thin-section CT findings. Radiology 1990;175(2):329-34. 